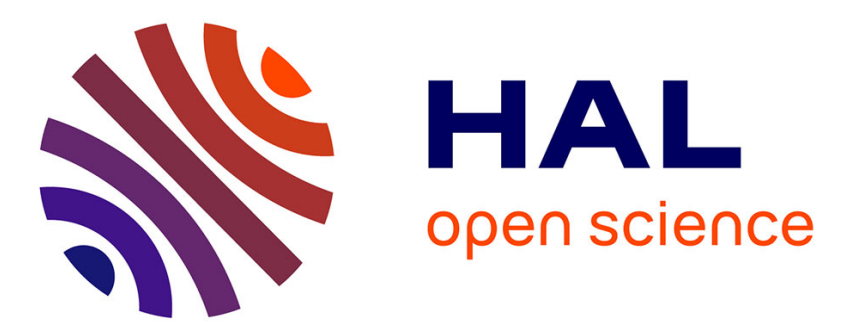

\title{
L'antibioprophylaxie en pratique courante en chirurgie urologique
}

\author{
E. Bey, M. Vallée, F. Saint, F. Bruyère
}

\section{To cite this version:}

E. Bey, M. Vallée, F. Saint, F. Bruyère. L'antibioprophylaxie en pratique courante en chirurgie urologique. Progrès en Urologie - FMC, 2019, 29, pp.F48 - F50. 10.1016/j.fpurol.2019.03.004 . hal03486362

\section{HAL Id: hal-03486362 \\ https://hal.science/hal-03486362}

Submitted on 20 Dec 2021

HAL is a multi-disciplinary open access archive for the deposit and dissemination of scientific research documents, whether they are published or not. The documents may come from teaching and research institutions in France or abroad, or from public or private research centers.
L'archive ouverte pluridisciplinaire HAL, est destinée au dépôt et à la diffusion de documents scientifiques de niveau recherche, publiés ou non, émanant des établissements d'enseignement et de recherche français ou étrangers, des laboratoires publics ou privés.

\section{다)(1) $(5$}

Distributed under a Creative Commons Attribution - NonCommerciall 4.0 International 


\title{
L'antibioprophylaxie en pratique courante en chirurgie urologique Antibiotic prophylaxis in daily practice in urological surgery
}

Elsa Bey1, Maxime Vallée2, Fabien Saint3, Franck Bruyère4 et les membres du CIAFU

\begin{abstract}
1 Service de chirurgie urologique, Centre Hospitalo-Universitaire de Grenoble, Grenoble, France 2 Service de chirurgie urologique, CHRU de Poitiers, 2 Rue de la Milétrie, 86021 Poitiers, France 3 Department. Urology and Transplantation, CHU Amiens Picardie, Amiens France

4 Service de chirurgie urologique, Centre Hospitalo-Universitaire de Tours, Tours, France

Auteur correspondant : Elsa Bey

EBEY@chu-grenoble.fr
\end{abstract}




\section{PRÉSENTATION DU CAS}

L'antibioprophylaxie ( $A B P$ ), qui fait pourtant l'objet de recommandations très claires émises par l'ensemble des sociétés savantes, est en pratique courante encore fréquemment un sujet de discorde entre urologues et anesthésistes, voire parfois un sujet de désintérêt mutuel pouvant amener à des pratiques à risque sur le plan de la prévention des infections associées aux soins. Nous avons souhaité faire le point sur cette thématique par le biais d'un cas clinique commenté que vous trouverez ci-dessous.

\section{QUESTIONS}

Question 1 - Vous arrivez au bloc opératoire au moment où entre votre patient prévu pour une résection endoscopique de prostate. L'ECBU pré-opératoire est stérile. A quel moment doit être réalisée l'injection d'antibiotique dans le cadre de son antibioprophylaxie?
a. Au moment de l'incision
b. N'importe quand au cours du geste
c. $\quad 30$ min avant l'incision
d. $\quad 2 \mathrm{~h}$ avant l'incision
e. Je ne sais pas

Question 2 - Quel antibiotique doit être administré ?
a. Amoxicilline -acide clavulanique $1 \mathrm{~g}$ dose unique
b. Tazocilline $4 \mathrm{~g}$ dose unique
c. Cefazoline $2 \mathrm{~g}$ dose unique
d. Cefuroxime $1,5 \mathrm{~g}$ dose unique
e. Amikacine $1 \mathrm{~g}$ dose unique

Question 3 - Votre patient est obèse, BMI > $35 \mathrm{~kg} / \mathrm{m} 2$. Cela modifie-t-il la dose de l'antibioprophylaxie?
a. Non
b. Oui, la dose doit être doublée.
c. Oui, la dose doit être augmentée de $25 \%$ pour les BMI > 30 et doublée pour les BMI $>35 \mathrm{~kg} / \mathrm{m} 2$.
d. Oui, la dose doit être augmentée de $50 \%$ pour les $\mathrm{BMI}>30$ et doublée pour les $\mathrm{BMI}$ $>35 \mathrm{~kg} / \mathrm{m} 2$.
e. Je ne sais pas

Question 4 - Faut-il faire une réinjection de l'antibiotique lors de l'intervention et si oui, au bout de combien de temps?
a. Non jamais.
b. Oui, toutes les heures à demi-dose.
c. Oui, au bout de quatre heures à demi-dose.
d. Oui, au bout de quatre heures à pleine dose.
e. Seulement en cas de facteurs de risques infectieux: patient immunodéprimé, diabétique ou sous corticothérapie.


Question 5 - Le patient était finalement déjà sous antibiotiques pour un ECBU pré-opératoire positif à Enterococcus faecalis. II prend de l'amoxicilline depuis $48 \mathrm{~h}$. Y a-t-il dans ce cas une indication à pratiquer une antibioprophylaxie et si oui, laquelle?

a. Non, il est déjà sous antibiothérapie.

b. Oui, on maintient l'antibioprophylaxie habituelle par Céfazoline ou Céfuroxime car son antibiothérapie était à spectre étroit.

c. Oui, on réalise une dose supplémentaire d'amoxicilline une demi-heure avant le geste.

d. Oui, on élargit le spectre et on réalise une injection de gentamicine avant l'induction.

e. Je fais ce que l'anesthésiste me dit de faire.

Question 6 - Votre patient est VIH+ sous trithérapie et greffé rénal. Faut-il dans ce cas modifier son antibioprophylaxie?

a. Non

b. Oui, il faut pratiquer une injection d'antibiotique large spectre de type TAZOCILLINE du fait de l'immunodépression.

c. Oui, il faut réaliser une association de 2 antibiotiques afin d'élargir le spectre.

d. Oui, je réalise d'ailleurs dans ce cas une antibiothérapie courte d'une semaine afin de prévenir le risque infectieux.

e. Je ne sais pas

Question 7 - Votre patient est porteur d'un SAMR dépisté sur écouvillon nasal lors d'une récente hospitalisation en gériatrie. Cela change-t-il votre attitude ?

a. Non

b. Oui, je fais pratiquer dans ce cas une injection de VANCOMYCINE avant l'incision.

c. Oui, je préfère faire pratiquer une antibioprophylaxie par CEFAZOLINE + VANCOMYCINE avant l'incision.

d. Je fais ce que l'anesthésiste me dit de faire.

e. Je ne sais pas

Question 8 - Pour quelles interventions suivantes réalisez-vous une antibioprophylaxie par Augmentin ${ }^{\circledR}$ ?

a. Pose de neuromodulateur des racines sacrées

b. Cure de prolapsus toutes voies d'abord

c. TOT/ TVT ou sphincter artificiel

d. Cystectomie

e. Chirurgie scrotale 


\section{RÉPONSES}

Réponse détaillée à la question 1 : $\mathbf{C}$.

Le moment de la prescription a fait l'objet d'un grand débat ces dernières années en particulier pour la chirurgie gynécologique. L'ABP doit toujours précéder l'intervention dans un délai d'environ 30 minutes. Ce point est fondamental. La séquence d'injection des produits d'induction doit être séparée de 5 à 10 minutes de celle de l'ABP, afin de faire la part de ce qui revient à chacune en cas de réaction allergique[1,2]. Les recommandations EAU 2018 vont même jusqu'à recommander un délai de 60 mn avant l'induction[3].

Une injection réalisée au moment de l'induction, comme c'est trop souvent le cas, est une mauvaise pratique : en effet, l'imprégnation tissulaire doit être suffisante au moment de l'incision. Elle doit donc théoriquement être réalisée soit en pré-anesthésie, soit dans le service AVANT la descente du patient au bloc opératoire.

\section{Réponse détaillée à la question 2 : C et $D$}

Accord entre les sociétés savantes (SFAR-AFU et EAU). En cas d'allergie, gentamicine $5 \mathrm{mg} / \mathrm{kg}$ dose unique. Les bactéries ciblées sont principalement les entérobactéries (Escherichia coli, Klebsiella, Proteus mirabilis...), Enterococcus et les staphylocoques (S. epidermidis surtout). Le choix de la molécule utilisée en antibiopophylaxie se base sur un cahier des charges précis :

- Celle-ci doit être active sur les microorganismes le plus souvent en cause dans les infections postopératoires tout en ayant le spectre le plus étroit possible

- Elle ne doit pas être, de préférence, celle habituellement utilisée en traitement curatif

- Elle doit exercer une moindre sélection de résistances bactériennes

- Tout en ayant une bonne pénétration dans le tissu cible.

Les fluoroquinolones n'ont pas de place pour l'ABP en chirurgie urologique (à l'exception des biopsies de la prostate), à la fois du fait de l'importance des résistances aux fluoroquinolones sur le territoire ( $15 \%$ pour E. coli et $27 \%$ pour K. pneumoniae en 2017 [4]) que de l'impact négatif de son utilisation en probabiliste sur l'émergence de résistances. Se référer aux recommandations de la SFAR 2017 mises à jour en juillet 2018 pour l'antibioprophylaxie recommandée pour chaque technique opératoire[1,2].

\section{Réponse détaillée à la question $3: \mathbf{B}$}

Pour les patients ayant un $\mathrm{BMI}>35 \mathrm{~kg} / \mathrm{m} 2$, une injection de Cefazoline de $4 \mathrm{~g}$ ou Cefuroxime $3 \mathrm{~g}$ doit être réalisée 30 minutes avant l'incision. L'obésité morbide représente à lui seul un facteur de risque d'infection du site opératoire (ISO). Par ailleurs, l'augmentation de la masse grasse impose, pour obtenir un volume de distribution de la molécule similaire à un sujet de poids standard, d'augmenter les posologies afin de maintenir une bonne diffusion tissulaire de la molécule. L'utilisation des traitements aux doses habituelles serait un facteur limitant au maintien d'un bon rapport PK/PD avec un risque important de sous dosages et donc d'inefficacité de l'antibioprophylaxie. Concernant les autres antibiotiques, se référer aux référentiels anesthésiques de chirurgie bariatrique.

\section{Réponse détaillée à la question $4: C$}

Une réinjection doit être réalisée toutes les 2 demi-vies, l'objectif étant d'avoir une concentration tissulaire efficace pendant tout l'acte opératoire (sauf aminoside). En urologie 
la plupart des interventions ont une durée inférieure à la demi-vie des molécules utilisées. La nécessité d'une réinjection dépend donc de l'antibiotique utilisé et de la durée de l'intervention chirurgicale. En pratique la Céfazoline et l'amoxicilline-acide clavulanique ont une demi-vie de $2 \mathrm{~h}$. Si le geste opératoire dure plus de $4 \mathrm{~h}$, il faut donc absolument penser à réaliser une nouvelle injection qui se fera cette fois à demi-dose de la première injection. En pratique clinique quotidienne, la cystectomie est donc habituellement la seule intervention chirurgicale où la réinjection antibiotique ne doit pas être oubliée!

\section{Réponse détaillée à la question 5 : B}

En cas d'antibiothérapie péri-opératoire à spectre étroit mise en place dans le cas du dépistage systématique d'une colonisation urinaire avant chirurgie urologique, une antibioprophylaxie classique par Céfazoline ou Céfuroxime doit être réalisée en sus afin de couvrir l'ensemble des espèces bactériennes habituellement rencontrées en urologie. En effet, dans cet exemple l'amoxicilline est la molécule de choix pour le traitement de l'Enterococcus feacalis mais a de très forts risques de ne pas être efficace sur la majeure partie des entérobactéries rencontrées en cas d'ISO. Par ailleurs, l'amoxicilline est rarement efficace sur le $S$. aureus si elle n'est pas associée à l'acide clavulanique. Les bactéries visées par une antibioprophylaxie par Cefazoline ou Cefuroxime sont donc bien plus nombreuses que celle traitée par une molécule à spectre étroit ciblée sur un antibiogramme précis. $\mathrm{Ne}$ pas réaliser l'antibioprophylaxie recommandée dans ce contexte revient donc à majorer le risque de sepsis postopératoire lié à des bactéries non " couvertes " par l'amoxicilline.

\section{Réponse détaillée à la question 6 : A}

Il n'existe pas d'indication à la modification de l'antibioprophylaxie en cas d'immunosuppression, quelle qu'elle soit. La flore de portage étant similaire à celle des sujets non immunodéprimés, le choix de l'antibiotique administré, ses modalités et sa durée d'administration restent les mêmes.

\section{Réponse détaillée à la question 7 : A}

Les recommandations restent floues sur ce point. La SFAR considère que le bénéfice pour le malade est dans ce cas probablement très faible par rapport à un surrisque infectieux mal évalué, d'autant plus que le SARM n'est pas un pathogène rencontré classiquement en urologie (en dehors de la pose de matériel). On rappelle à ce titre qu'en urologie, le dépistage pré-opératoire de SARM n'a actuellement pas d'indication.

Ceci peut être discuté entre l'anesthésiste et le chirurgien avant l'intervention. On recommande habituellement de ne pas tenir compte de la flore de portage (sauf bien sûr s'il s'agit d'un portage urinaire) dans le choix de l'antibioprophylaxie à administrer.

\section{Réponse détaillée à la question $8: A, B, C, D$}

Toutes les propositions sauf la chirurgie scrotale, sauf si mise en place de prothèse. Si une prothèse doit être posée, l'antibioprophylaxie à réaliser est une injection de Cefazoline $2 \mathrm{~g}$ dose unique, avec réinjection à demi-dose si durée $>2 \mathrm{~h}$. Le choix d'une bêtalactamine associée à de l'acide clavulanique s'explique par la nécessité de couverture du SAMS et des anaérobies, fréquemment rencontrés dans les infections sur matériel prothétique. 


\section{Références}

1. Mise à jour de la RFE Antibioprophylaxie 2017 - La SFAR. Société Française d'Anesthésie et de Réanimation. https://sfar.org/mise-a-jour-de-la-rfe-antibioprophylaxie-2017/. Published July 5, 2018.

2. Antibioprophylaxie en chirurgie et médecine interventionnelle (patients adultes) - La SFAR. Société Française d'Anesthésie et de Réanimation.

https://sfar.org/antibioprophylaxie-en-chirurgie-et-medecine-interventionnellepatients-adultes-2017/. Published August 29, 2018.

3. Professionals S-O. Urological Infections. Uroweb. http://uroweb.org/guideline/urological-infections/

4. http://atlas.ecdc.europa.eu/public/index.aspx 


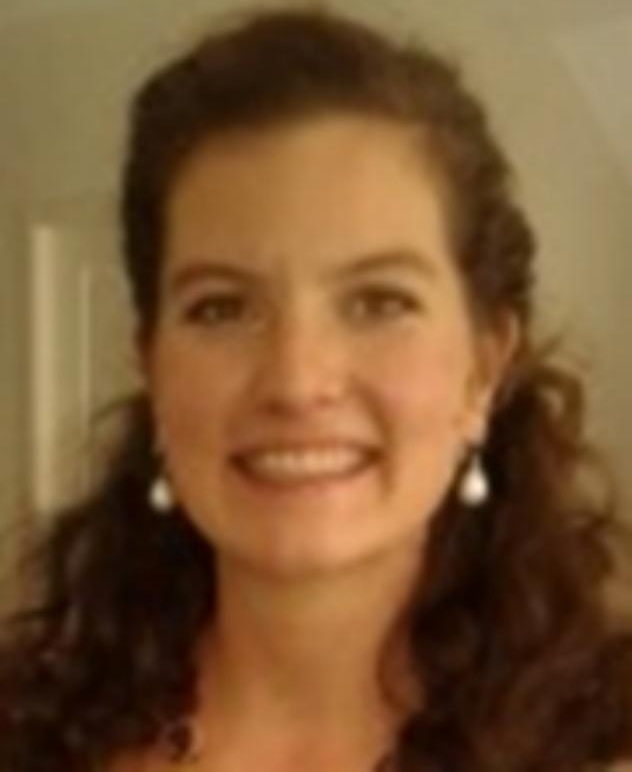

